\title{
The Identification of Electric Load Simulator for Gun Control Systems Based on Variable-structure WNN with Adaptive Differential Evolution
}

\author{
Wang Chao", Hou Yuanlong, Liu Rongzhong, Gao Qiang and Hou Runmin \\ School of Mechanical Engineering, Nanjing University of Science and Technology, Nanjing 210094, China.
}

\begin{abstract}
Owing to the complex nonlinearities of the electric load simulator (ELS) for the gun control system (GCS), the surplus torque plays a great negative impact on the performance of the loading system. This paper proposes a variable-structure wavelet-neural-network (VSWNN) identification strategy based on adaptive differential evolution (ADE). First of all, a mathematical model is established based on the structure and the working principle of the ELS. Then an intelligent identification method is applied, where the wavelet function is chosen as the excitation function, which improves the generalization and approximation ability of the neural network. The ADE is used to optimize the parameters, which solves the difficulty of determining the structure of the WNN. In order to reduce the computation complexity and speed up the convergence of the identification system, the adaptive laws of the pitch adjusting rate (PAR), band width (BW) and variable numbers of neurons are proposed. Finally, a pseudo random multilevel signal and a linear frequency modulation signal are chosen as input signals for the hardware-in-the-loop simulation. The test results show that the proposed ADE-VSWNN algorithm has superior validity and practicability, especially when the identification algorithm is used in the working circumstances with different inertial torque. Further, the high precision and strong robustness of the identification algorithm are further verified.
\end{abstract}

Keywords: Electric load simulator; Gun control system; Adaptive differential evolution; Variable-structure WNN.

\section{Introduction}

In order to further improve the control precision and the dynamic response performance of GCSs, load simulators are commonly chosen as the hardware-in-the-loop simulation test platform for the GCS. Naturally, the control performance and the anti-interference capability of load simulators directly affects the quality of the GCS. According to the differences in drive types, the load simulators are divided into electrohydraulic load simulators and electric load simulators. The former are applied to heavy loads [1-6]; the latter, being characterized by small noise, low environmental pollution, high reliability, easy maintenance, etc., are widely used in relatively light loading conditions. The steering engines in aircraft have been mostly and deeply studied recently [1-6]. However, the research projects on load simulators for GCSs proposed in this paper are relatively few $[7,8]$.

To further improve the control precision of ELSs, the internal causes leading to nonlinearities, such as friction, backlash, inertia, etc., should be fully studied. In view of the problems caused by the friction being nonlinear, research scholars have increasingly adopted compensation schemes to solve them. In [9-10], scholars establish a mathematical model with the friction model. Under the condition of system stability, measurement conditions are estimated based on a state observer that compensates for the friction nonlinearity in the system. In [11], the focus is on the friction at low speed. Considering the friction influence on the performance of the system, the relative adaptive criterions that can effectively limit the negative influence of friction are established. In addition, the backlash nonlinearity also plays a great negative effect on the system. To eliminate the existing backlash in the system in [12], a frequency identification with continuous estimate based on sinusoidal excitation and analytic geometry is designed, which effectively forecasts the frequency response function and the backlash of the nonlinear boundary when the system is working in a specified area and frequency. In [13-14], the emphasis is placed on the backlash and inertia nonlinearities, which do harm to the gear dynamic movement. Furthermore, multiple degrees of freedom systems with different vibration phenomena are studied, and a stability analysis based on the multi-scale method is performed to establish a dynamic self-tuning backlash motion model.

In order to make the ELS possess a stable, fast and accurate tracking response to the reference command, using the traditional control algorithms (such as PID and adaptive PID, etc.) is hard to achieve ideal identification and control [7]. However, efficient intelligent algorithms, which were better fitted to the characteristics of the ELS [4-9], have emerged at the right moment. Methods based on neural networks are effective when applied to the identification of dynamic nonlinear systems, which are also effective in combinatorial optimization [15], optimization of non-stationary functions [16] multi-objective optimization [17], bioinformatics [18]. The WNN with the merits of local optimization, approximation and self-learning in the time-frequency domain provides a framework that can be successfully applied to the dynamic models [19-22] and the nonlinearity control [23-26]. In [19], the wavelet representation with high-efficiency coupled map lattices trains the basic structure of the WNN, and a novel two-stage hybrid training scheme is developed for constructing a parsimonious WNN to improve the identification efficiency. A differential evolution algorithm in [20] and a storage strategy in [21] are used to optimize the WNN parameters in various ways, which guarantees the performance and robustness of the system in the sense of Lyapunov stability.

In this paper, the identification method of the ADE-VSWNN is proposed. The crossover and mutation operation, which optimizes the scale factors, the translation factors, and the weights of the WNN [20] is realized by using the individual genetic difference algorithm. The DE algorithm has the advantages of fast speed, strong robustness and wide search ability in the real domain, but its search performance is affected by the individual variations. If the mutation rate is too large, the optimal solution will be damaged and very difficult to be found. The mutation rate is too small to guarantee the diversity of population, which can also easily fall into a local optimal solution [27]. As a consequence, an adaptive crossover and a mutation rate adjusted by the search algorithm are designed, which can maintain the diversity of population and avoid the premature convergence of the algorithm. Considering the identification method applied to a real-time system, the actual 
computation efficiency and convergence speed must be appropriate. A characteristic evaluation coefficient for the hidden layer is built, which involves the scale factors and the translation factors of the wavelet function and dynamically adjusts the actual number of neurons [21, 25]. In addition, the variable-structure WNN shows great merits applied to real-time nonlinear systems [28-31], and favorable control performance can be achieved.

This paper is organized as follows. A brief introduction to the composition and working principles of the ELS is given in Section 2. In Section 3, taking into account the nonlinearities existing in the system, the mathematical model of the ELS for GCSs is established. The proposed ADE-VSWNN identification is presented in detail in Section 4. In Section 5 effective simulation tests in various conditions, as well as a comparative analysis, verify the identification method effectively and precisely.

\section{Composition and working principle}

The ELS for GCSs is mainly composed of a host control computer, a signal conditioning center, a torque motor, a motor drive, a torque sensor, a resolver, an angular velocity sensor, an angular acceleration sensor, inertia disks and a gear reducer, etc., shown in Fig.1. The host control computer with the proposed algorithm to achieve torque tracking control of the ELS mainly analyzes and calculates the feedback torque of the torque sensor, the compensation torque of inertia disks, and the actual torque required. The signal conditioning center is mainly used for processing signals from the sensors and the configuration settings needed for the hardware switches. According to the output of the host control computer, the output of drive provides the appropriate current to drive the torque motor. The torque sensor is used to collect the output of the torque motor, which is fed back to the host control computer to form the closed-loop control for the loading torque. The function of the resolver is gathering the angle position of the gear reducer, which can be used to calculate the real-time command torque for the torque motor. The function of the angular velocity sensor is gathering the angular velocity of the gear reducer to provide more actual values for the identification methods and the control strategies. The angular acceleration sensor collects the angular acceleration at the end of the gear reducer to compute the inertia moment produced by the inertia disks. The number of inertia disks depends on the actual demand of the GCS, which provides various rotational inertia values to simulate the inertia torque.

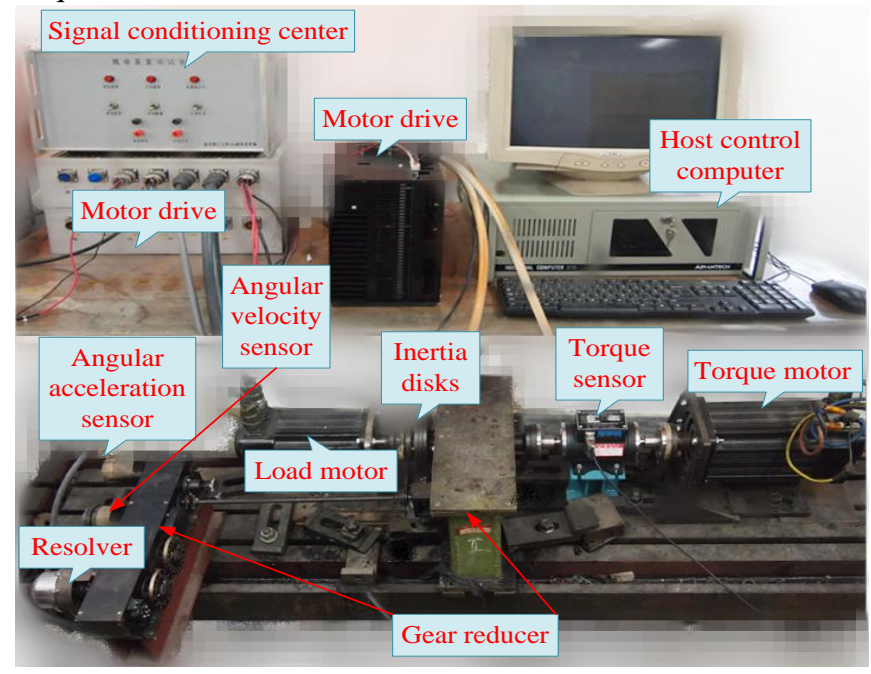

Fig. 1. The composition diagram of the ELS for GCSs
In order to make the ELS conform to the real-time changing torque and to take the rapidity and stability into account, a permanent magnet AC synchronous motor (PMACSM) working in torque mode is chosen as torque motor [32], which simulates the resistance torque on the basis of the command torque existing in the GCS (including friction torque and gravitational torque). On account of the variation of the gun barrel angle and the number of shots when launching, inertia disks are adopted to simulate the inertia torque so that the surplus torque is largely reduced to improve the performance and robustness of the ELS control, which is different from the steering ELS focused on the resistance torque caused by wind and airflow [33-34]. Considering the examination of the identification and control accuracy in the GCS, a load motor (also named as position motor) applied to position control is usually added to the other side of the gear reducer, which is made to work in the process of rotating the gun barrels and to simulate the real work situation of the ELS.

\section{Mathematical model with nonlinearities}

The torque motor of the ELS is a PMACSM with high efficiency, high torque of inertia ratio, fast response and high precision, the control current and output torque of which are not in a simple linear relationship compared with the direct loading torque motor. In order to solve the problem of the self-coupling of the PMACSM, the motor uses a vector control oriented magnetic field [32], which allows variables like voltage, current and magnetic potential be decoupled. In order to further analyse problems, the simplified motor model on the basis of taking the characteristics of the GCS into account and ignoring secondary factors, the PMACSM can be approximately expressed as a model of DC torque motor in which the quadrature axis current is zero. In terms of working principle, basic structure and characteristics, the DC torque motor is familiar with a DC brushless servo motor, so the armature circuit voltage balance equation is as follows

$$
u_{m}(t)=R_{m} i_{m}(t)+L_{m} \frac{d i_{m}(t)}{d t}+E_{m}
$$

where $u_{m}(t)$ is the armature voltage; $R_{m}$ is the equivalent resistance of the armature circuit; $i_{m}(t)$ is the armature current; $L_{m}$ is the equivalent inductance of the armature circuit; $E_{m}$ is the armature electromotive force, which is produced when the armature rotates and proportional to the excitation magnetic flux and speed.

The counter electromotive force can be written as follows

$$
E_{m}=K_{e} \omega_{m}(t)=K_{e} \frac{d \theta_{m}(t)}{d t}
$$

where $K_{e}$ is the coefficient of counter electromotive force; $\omega_{m}(t)$ and $\theta_{m}(t)$ are the angular velocity and angular position of the torque motor rotor, respectively. The torque of the motor balance equation is

$$
T_{m}=K_{t} i_{m}=J_{m} \frac{d \omega_{m}(t)}{d t}+B_{m} \omega_{m}(t)+T_{n}(t)
$$

where $T_{m}$ is the electromagnetic torque; $K_{t}$ is the coefficient of electromagnetic torque; $J_{m}$ is the rotational inertia converted to the torque motor shaft; $B_{m}$ is the viscous friction coefficient of the motor rotor; $T_{n}(t)$ is the interference torque on the torque motor shaft.

The causes of the interference torque of motor shaft are mainly focused on friction, backlash, inertia, which do help to 
establish the mathematical model of mechanism of the GCS ELS. The friction between the motor and the gear reducer existing in the transmission mechanism is very complex when the working situation (such as temperature, lubrication and wear condition) varies irregularly. Especially when the motor is working at a low speed, the changes of the friction have a great effect on the performance of the ELS. The LuGre friction model is widely used in the ELS [9-11]. Aiming at characteristics of a different ELS system itself, a dynamic LuGre friction model is built [35], which can be expressed as follows

$$
\begin{aligned}
& \frac{d z}{d t}=\omega_{a}^{\prime}-\frac{\left|\omega_{a}^{\prime}\right|}{g\left(\omega_{a}^{\prime}\right)} z \quad \omega_{a}^{\prime}=\omega_{m} / i_{g} \\
& \sigma_{0} g\left(\omega_{a}^{\prime}\right)=F_{c}+\left(F_{s}-F_{c}\right) \cdot e^{-\left(\frac{\omega_{a}^{\prime}}{\omega_{s}}\right)^{2}} \omega_{s} \\
& T_{b f}=\sigma_{0} z+\sigma_{1} \frac{d z}{d t}+\sigma_{2} \omega_{a}^{\prime}
\end{aligned}
$$

where $z$ is the friction state that physically stands for the average deflection of the bristles between two contact surfaces; $\omega_{a}^{\prime}$ is the rotor angular velocity through the gear reducer; $i_{g}$ is reduction ratio; the nonlinear function $g\left(\omega_{a}^{\prime}\right)>0$ that stands for a different friction effect [36]; $F_{c}$ is the Coulomb friction; $F_{s}$ is the largest static friction torque; $\omega_{s}$ is the Stribeck speed; $T_{b f}$ is the friction torque; $\sigma_{0}, \sigma_{1}$ and $\sigma_{2}$ are the friction force parameters which can be physically interpreted as the stiffness of the bristles between two contact surfaces and the damping coefficient of the bristles. Considering factors like temperature, lubrication condition and wear, the friction coefficient $\lambda$ is introduced to reflect the change trend of the friction torque [37], where under normal working conditions, the friction torque of the system is standard. When the parameters of LuGre model change, the friction torque will change, which can be reflected through $\lambda$. Taking these into account, the friction torque can be defined as

$$
\begin{aligned}
T_{b f} & =\lambda\left(\sigma_{0} z+\sigma_{1} \frac{d z}{d t}+\sigma_{2} \omega_{a}^{\prime}\right) \\
& =\lambda\left(\sigma_{0} z+\sigma_{1}\left(\begin{array}{c}
\left.\left.\omega_{a}^{\prime}-\frac{\sigma_{0}\left|\omega_{a}^{\prime}\right|}{F_{c}+\left(F_{s}-F_{c}\right) \cdot e^{-\left(\frac{\omega_{a}^{\prime}}{\omega_{s}}\right)^{2}}} z\right)+\sigma_{2} \omega_{a}^{\prime}\right)
\end{array}\right)\right.
\end{aligned}
$$

The nonlinear backlash existing between the gear reducer and the flexible coupling gaps in the ELS is seen as indispensable for the ELS. It has a great influence on the dynamic performance and steadiness of the accuracy of the system [12-14]. In addition, the rigid collision of gears will produce serious vibration and noise [38]. Hence, it is of great theoretical significance and engineering practical worth to perform in-depth research on the backlash nonlinearity existing in the system. The backlash disturbance torque can be obtained as follows

$$
T_{b b}=K_{b} b(t)
$$

where $K_{b}$ is the torque coefficient of backlash; $b(t)$ is a dead-zone function, defined as follows

$$
b(\delta)=\left\{\begin{array}{cc}
\delta+\alpha, & \delta<-\alpha \\
0, & |\delta| \leq \alpha \\
\delta-\alpha, & \delta>\alpha
\end{array}\right.
$$

where $\alpha$ is the width of backlash; $\delta=\theta_{m}-i_{g} \theta_{a}^{\prime}$ is the relative angular displacement between the torque motor shaft and the load side; $\theta_{a}{ }^{\prime}$ is the angular displacement of load side. The design of the controller is not easy to perform because of the non-differentiable features of the dead-zone function. Thus, the continuous approximation dead-zone function is introduced [39]

$$
b^{*}(\delta)=\delta-c \alpha\left(\frac{2}{1+e^{-r \delta}}-1\right)
$$

where $a>0$ and $r>0$ are undetermined parameters. In order to analyze the degree of approximation to the dead zone-function, the difference between the continuous approximation dead-zone function and the dead-zone function is defined as follows

$$
\Delta b(\delta)= \begin{cases}-c \alpha\left(\frac{2}{1+e^{-r \delta}}-1\right)-\alpha, & \delta<-\alpha \\ \delta-c \alpha\left(\frac{2}{1+e^{-r \delta}}-1\right), & |\delta| \leq \alpha \\ -c \alpha\left(\frac{2}{1+e^{-r \delta}}-1\right)+\alpha, & \delta>\alpha\end{cases}
$$

According to the formulas above, when the parameter $a$ equals 1 and $r$ equals $2 / \alpha$, the following conclusions can be achieved: 1) $\left.\lim _{\delta \rightarrow \infty} \Delta b(\delta)=0 ; 2\right) b^{*}(\delta)$ is a monotonic increasing function; 3) The area between $b^{*}(\delta)$ and $b(\delta)$ is the least; 4) $|\Delta b(\delta)| \leq \frac{2 \alpha e^{-r \alpha}}{1+e^{-r \alpha}}$.

The influence that inertia disks have on the ELS appears mainly in the case of the variations in the motor speed, when the motor acceleration is not zero, which results in a corresponding inertia torque. Due to the inertia disks installed in the center of torque motor output shaft, and the number of corresponding inertia disks is variable, the inertia torque can be obtained as follows

$$
T_{i}=K_{j} J_{i} \frac{d \omega_{m}(t)}{d t}
$$

where $K_{j}$ is the coefficient of inertia torque; $J_{i}$ is the inertia of inertia disks.

A torque sensor is placed in the middle of the torque and position motor, with the main purpose of detecting the angular deviation between two motor shafts. Thus the torque under working conditions is

$$
T_{g}=G_{f}\left(\theta_{m}-\theta_{a}\right)
$$

where $G_{f}$ is the elastic coefficient of the torque sensor and $\theta_{a}$ is the angular position of the rotor of the position motor. To sum up, when ignoring the other nonlinearities with negative influences, the structure diagram of the ELS for GCSs can be obtained as shown in Fig. 2. The interference torque on the torque motor shaft can be written as

$$
T_{n}=T_{i}+T_{g}+T_{b f}+T_{b b}
$$

where $T_{b}=T_{b f}+T_{b b} ; K_{r}$ is the feedback coefficient of torque loop; $K_{l}$ is the feedback constant of the motor current; $K_{u}$ is the voltage gain of the motor drive; and $K_{i}$ is the current 
gain of the motor drive.

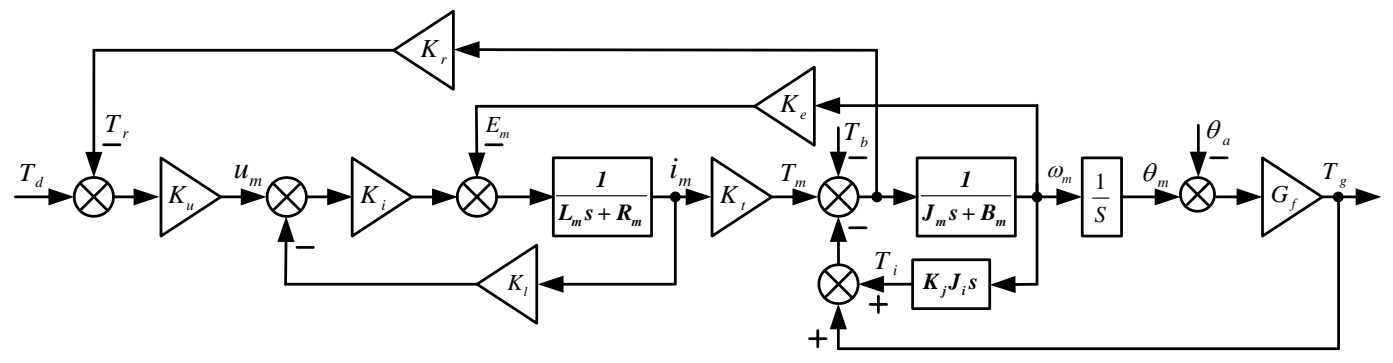

Fig. 2. The structure diagram of the ELS for GCS

As shown in Fig. 2, the value of the torque sensor gathered is set to be a controlled variable, $T_{g}$ is the output of the system, while $T_{d}, T_{b}$ and $\theta_{a}$ are chosen as the inputs of the system. According to the superposition theorem, we can obtain the following transfer function

$$
\begin{aligned}
& T_{g}= \frac{K_{t} K_{u} K_{i} G_{f}}{\left(K_{j} J_{i} s^{2}+Q_{1} s+G_{f}\right) Q_{2}+\left[K_{e} K_{t}+K_{t} K_{u} K_{i} K_{r} Q_{1}\right] s} T_{d} \\
&-\frac{G_{f}\left[\left(Q_{1}+K_{j} J_{i} s\right) Q_{2} s+K_{t}\left(K_{u} K_{r} Q_{1} s+K_{e}\right)\right]}{\left(Q_{1} s+K_{j} J_{i} s^{2}-G_{f}\right) Q_{2}+K_{t}\left(K_{u} K_{r} K_{i} Q_{1} s+K_{e}\right)} \theta_{a} \\
&+\frac{G_{f} Q_{2}}{K_{t}\left(K_{u} K_{i} K_{r} Q_{1}+K_{e}\right) s+\left(Q_{1} s-K_{j} J_{i} s^{2}-G_{f}\right) Q_{2}} T_{b} \\
& \text { where } Q_{1}=J_{m} s+B_{m}, Q_{2}=L_{m} s+R_{m}+K_{i} K_{l} .
\end{aligned}
$$

In (15), the torque value $T_{g}$ consists of three parts. The first part can be seen as being related to the control signals of the initiative loading torque (reference command torque). The second part can be seen as being related to a passive load caused by the position motor, when position disturbance $\theta_{a}$ equals zero, which means that the output position of the load side is fixed. Then, the torque motor is in the blocked state, and the output torque $T_{g}$ can be obtained by setting the torque $T_{d}$ in this control mode. The third part, termed interference torque, results mainly from friction, backlash and other uncertainties. $J_{m}, B_{m}, L_{m}, R_{m}$ are variable in real-time working conditions. The causes are comparatively complex and showing strong nonlinear characteristics, which make it difficult to establish an exact mechanism model. Thus, the following intelligent control has been a popular trend to solve the nonlinearities.

\section{Variable-structure WNN with adaptive differential evolution algorithm}

\subsection{WNN}

The term wavelet means a little wave which is special in that a continuous wavelet transformation can be regarded as a flexible time-frequency window. The narrower the window is, the higher the frequency of signals is, and vice versa. Wavelet transforms can be divided into continuous wavelet transforms and discrete wavelet transforms; the corresponding forms are below

$$
\begin{aligned}
& C W T(a, b)=\frac{1}{\sqrt{a}} \int x(t) \Psi\left(\frac{t-b}{a}\right) \\
& \operatorname{DWT}(a, b)=\sum_{i} x(t) a_{i}^{-1 / 2} \Psi\left(\frac{t-b_{i}}{a_{i}}\right)
\end{aligned}
$$

where $\Psi$ is a wavelet function, while $a$ and $b$ are the scale and translation factor of the wavelet function, respectively.
Considering the limitations of the continuous wavelet transform in applications related to digital computing, the discrete wavelet transform is the transform usually applied in practical engineering. Many times, a wavelet transform with advantages of local time and frequency domain is combined with a neural network, which possesses the ability of strong self-learning, approximation and fault-tolerance. Thus, the wavelet neural network is widely used in the field of the pattern recognition [40], system identification [19-22], system control [23-26], etc. Generally, a WNN consists of three layers, which includes the input layer, the hidden layer and the output layer, as shown in Fig.3.

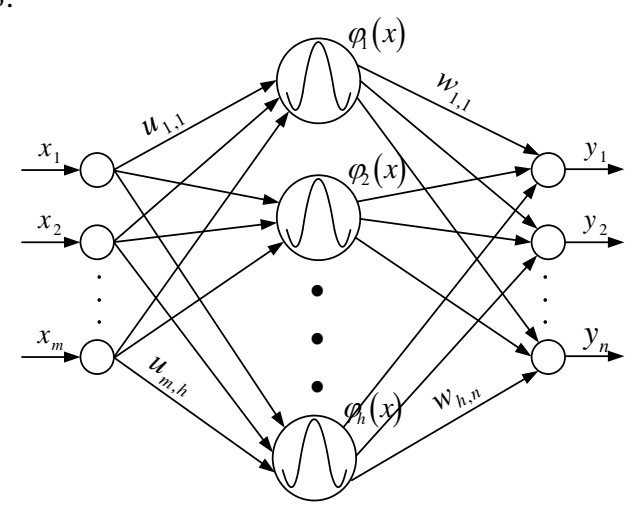

Fig. 3. The structure diagram of WNN

Taking into the characteristics of the object needed be identified consideration, a Morlet wavelet function is chosen as the excitation function of the wavelet node [41]

$\varphi_{i}(x)=\cos \left(1.75 \times \frac{x-b_{i}}{a_{i}}\right) \exp \left(-\frac{\left(x-b_{i}\right)^{2}}{2 a_{i}}\right) \quad i=1,2,3, \ldots, m$.

Then, the output of the wavelet neural network is as follows

$y_{j}=\sum_{k=1}^{h} w_{k, n} \varphi_{k}\left(\sum_{i=1}^{m} u_{i, k} x_{i}\right) \quad j=1,2, \ldots, n$.

where $a_{i}$ and $b_{i}$ are respectively the scale and the translation factor of the wavelet function at the i-th node; $x_{i}$ is the input of the $i$ th node in the input layer; $y_{j}$ is the output of the $\mathrm{i}$-th node in the output layer; $u_{i, k}$ is the weight between the $\mathrm{i}$-th node in the input layer and the $k$ th node in the hidden layer; $w_{k, j}$ is the weight between the $k$ th node in the hidden layer and the $k$ th node in the output layer; $m$ is the number of nodes in the input layer; $h$ is the number of nodes in the hidden layer; $n$ is the number of nodes in the output layer; $\varphi_{i}$ is the basis of the wavelet which is obtained by scaling and translating the mother wavelet $\psi(x)=\cos (1.75 x) \exp \left(-x^{2} / 2\right)$.

\subsection{Adaptive differential evolution algorithm}

Many optimization algorithms are proposed for increasing search ability and improving the convergence rate, such as the 
differential evolution (DE) [42], Hooke and Jeeves (HJ) algorithm [43], particle swarm optimization algorithm [44], gradient descent algorithm [25, 29], et al.. The differential evolution algorithm was proposed by Storn and Price in 1995. It is a kind of random search algorithm based on the new groups and able to handle the optimization problems of nondifferentiable, nonlinear and multi-modal objective functions. The DE with Hooke and Jeeves local search method is developed based on a genetic algorithm, which makes use of an individual difference genetic algorithm to implement the crossover and mutation operation [44-45]. The researchers focus on seeking a global optimum relying on the superior harmony. The harmony memory with size (HMS) is initially populated with randomly solutions sorted by corresponding objective function values. Thus, many adaptive differential evolution (ADE) algorithms are developed for optimizing mixed discrete continuous engineering problems based on the harmony considering rate (HMCR) and the pitch adjusting rate (PAR) [44-45]. In addition, we employ the adaptive harmony search (HS) proposed by Mahdavi et al. by adaptive two parameters: PAR and band width (BW) [46]. PAR and BW with each improvisation step $t$ are defined as follows:

$$
\begin{aligned}
& P A R(t)=P A R_{\min }+\frac{P A R_{\max }-P A R_{\min }}{N I} \cdot t \\
& B W(t)=B W_{\max } \cdot \exp \left(t \cdot \frac{L n\left(B W_{\min } / B W_{\max }\right)}{N I}\right)
\end{aligned}
$$

where $N I, B W_{\min }, B W_{\max }, P A R_{\min }$ and $P A R_{\max }$ are the number of improvisations, minimum/maximum pitch adjusting rate/bandwidth, respectively. The HJ local search is used to exploit the candidate solutions if they are randomly selected based on a pre-specified percentage, $p=0.1$. The pseudo codes of the ADE are in the following.

(1) Specify the DE-related parameters, including $N P, F, C R$ and Maxnfe. Specify HS-related parameters, including HMS, NI, $H M C R, B W_{\min }, B W_{\max }, P A R_{\min }$ and $P A R_{\max } . S e t H M S$ and $N P$ to be equal. Specify HJ-related parameters, including $\lambda, M_{\max }$ and $p$. Set the number of function evaluations ( $n f e$ ) equal to $N P$. The Table 1 summarizes the settings of all relevant algorithmic parameters of the $\mathrm{ADE}$ based on the previous experiments and studies. Give the upper/lower bound of each parameter. The parameters above and the parameters of WNN are given optimal initial values offline based on the previous experiments.

(2) According to the purpose of the research, a global optimum value of the appropriate fitness function is chosen.

(3) Handle constraints by using the Deb's method, and sort feasible and infeasible solutions according to Deb's constraint handling method. Retain the top 50\% solutions in the HM and as the initial solutions of DE.

(4) While $n f e<$ Maxnfe and $f_{\text {best }}<0.999$

For each target vector $(=1,2, \ldots, N P)$

Generate a mutated and trial vector based on the DE algorithm and handle bound violations.

Construct a new HS solution based on the HS algorithm.

Update the PAR and $B W$ based on the Eq. (20) and (21).

If rand $<H M C R$

Randomly pick a value form the HM.

If rand $<P A R$

Adjust the pitch for improving the harmony $v^{\prime}=v^{\prime} \pm$ rand $\cdot B W$ Check and repair the bound violations.

Else End if

Randomly generate a value within the domain range. End If

If the new solutions are randomly selected (with a specified percentage, $p$ )

Apply HJ (presented above) and increment $n f e$ accordingly Else

Evaluate the new solutions found by both algorithms and increment $n f e$ by two.

End if

Replace the target vector with the new trail vector for the DE algorithm if there is an improvement, and update the HM.

Update the best solutions.

End for

End while

Print output

Table 1

The global algorithmic parameters of the ADE

\begin{tabular}{cc}
\hline Index & Value \\
\hline Population size & $N P=3 \times m \times h+h \times n$ \\
Scale factor & $F=0.5$ \\
Crossover rate & $C R=0.90$ \\
Harmony memory size & $H M S=3 \times m \times h+h \times n$ \\
Harmony considering rate & $H M C R=0.95$ \\
Number of improvisations & $N I=$ Maxnfe $=100$ \\
Minimum pitch adjusting rate & $P A R_{\min }=0.5$ \\
Maximum pitch adjusting rate & $P A R_{\max }=0.95$ \\
Minimum bandwidth & $B W_{\min }=0.001$ \\
Maximum bandwidth & $B W_{\max }=3.0$ \\
Stepsize & $\lambda=0.1$ \\
Maximum number of iterations & $M_{\max }=10$ \\
Probability to perform HJ local search & $p=0.1$ \\
\hline
\end{tabular}

\subsection{Variable-structure WNN with the ADE}

The scale, translation and the weight factors of WNN are described as chromosomes when the ADE is in the learning process of optimizing the WNN. The appropriate fitness function is chosen, which makes the WNN rapidly converge to the optimal value by the steps of the ADE above.

\subsubsection{Genetic algorithm encoding}

The encoding methods for the genetic algorithm chromosomes mainly include binary encoding and real number encoding. If the binary encoding is adopted, all parameters need to be converted to binary numbers, which results in low efficiency and a complex computation for the learning algorithm. Moreover, those binary numbers need to be decoded to real numbers at the end. To improve the computational efficiency, the real number encoding method is used to design the encoding scheme as shown in Fig. 4. The Fig. 4 takes for example the $k$ th neuron in the hidden layer of WNN. In this figure, $u_{1, k}, \ldots, u_{m, k}$ are the weights between the input and the hidden layer; $w_{k, 1}, \ldots, w_{k, n}$ are the weights between the hidden and the output layer; and $a_{k}$ and $b_{k}$ are the scale and translation factors of WNN at the k-th node.

\begin{tabular}{|l|l|l|l|l|l|l|l|l|l|}
\hline$\cdots$ & $u_{1, k}$ & $\cdots$ & $u_{m, k}$ & $w_{k, 1}$ & $\cdots$ & $w_{k, n}$ & $a_{k}$ & $b_{k}$ & $\cdots$ \\
\hline
\end{tabular}

Fig. 4. The chromosome encoding of WNN

\subsubsection{Fitness objective function}

To effectively evaluate the parameters of the WNN in order to establish the identification accuracy of the neural network, the identification error function $\mathrm{E}$ of the $\mathrm{WNN}$ is chosen to be the fitness function of a dependent variable. Generally, the greater the value of the fitness function, the better the effect of identification. At the same time, in order to avoid an absolute error which is too small, the fitness function of adaptive differential evolution of the $\mathrm{WNN}$ is as follows 


$$
f=\frac{1}{1+E}
$$

where $E=\frac{1}{n} \sum_{j=1}^{n}\left(y_{j}-\hat{y}_{j}\right)^{2}, \quad y_{j}$ is the actual output of the system, and $\hat{y}_{j}$ is the output of ADE-WNN. When the $E$ is at the minimum, $f$ is at its maximum, and the structural parameters of the ADE-WNN are the most optimal.

\subsubsection{Variable-structure algorithm}

In order to reduce the computational complexity and improve the convergence speed, the method of dynamically adjusting the structure of the neural network is adopted to alleviate the contradiction between the computational complexity and the ability to approximate [28-31, 47]. This paper proposes an online network self-evolving scheme using a Mahalanobis distance (M-distance). The M-distance between the input variable and the existing hidden neurons is defined as

$$
M_{i}=\sqrt{\frac{\left(x-b_{i}\right)^{2}}{a_{i}^{2}}} \quad i=1,2, \cdots, m \text {. }
$$

If the new input variable calculated falls within the scope of the existing hidden neurons, it is necessary to reduce the M-distance. As a result, the ADE-WNN will not generate new neurons but update the parameters of the existing hidden neurons. According to the traditional method of variablestructure, a new hidden neuron is generated when a new input variable is too far away from the current clusters. The criterion of generating a new hidden neuron for new incoming data is described and the minimum distance is defined as [47]

$$
M_{\min }=\min _{1 \leq i \leq n} M_{i}
$$

If $M_{\min }>M_{0}$ is satisfied, a new neuron is added, where $M_{0}$ is a pre-given positive constant. It is known that the smaller the $M_{0}$, the easier the new neurons are produced. The new hidden neuron parameters are as follows

$$
\left\{\begin{array}{l}
b_{\text {new }}=x_{0} \\
a_{\text {new }}=\bar{a} \\
u_{\text {new }}=0
\end{array}\right.
$$

where $b_{\text {new }}, a_{\text {new }}$ and $u_{\text {new }}$ are the scale, translation and weight factors of the new neuron, respectively, while $x_{0}$ and $\bar{a}$ are pre-specified constants.

The number of new neurons at the $\mathrm{k}$-th sample period is increased as

$$
h(k+1)=h(k)+1
$$

To avoid the structure of ADE-WNN growing unboundedly, a pruning algorithm based on the density is introduced in this method to prune the inappropriate hidden neurons. An influence index is taken as the importance of neurons, which is defined as

$$
G_{j}(k+1)=\left\{\begin{array}{cc}
G_{j}(k) \exp (-\tau) & M_{j}>\theta \\
G_{j}(k) & M_{j} \leq \theta
\end{array} \quad j=1,2, \cdots, h .\right.
$$

where $G_{j}(k)$ is the influence index of the $\mathrm{j}$-th hidden neuron at the $\mathrm{k}$-th sample period, the initial value being $1 ; \theta$ is a descent threshold constant; $\tau$ is a speed constant.

If $G_{j}(k) \leq G_{0}$ is satisfied, where $G_{0}$ is the pre-given positive constant, the $\mathrm{j}$-th neuron is eliminated. In order to realize an effective control of real-time systems, the number of neurons must be reduced to simplify the computational complexity, and the selection of $G_{0}$ is 0.68 . Of course, the larger the number of neurons, the better the identification performance and the slower the convergence.

\subsection{The summary of this section}

Synthesizing these algorithms above, first, the actual input and output preprocessed of the ELS need be determined. Second, taking the preset threshold optimization of the WNN structure into consideration, the parameters of the WNN are preliminarily determined. Third, the adaptive differential evolution algorithm is adopted to train parameters of the variable-structure WNN (VSWNN). Finally, the optimized network (ADE-VSWNN) is used to identify the ELS system. The identification scheme is as shown in Fig. 5.

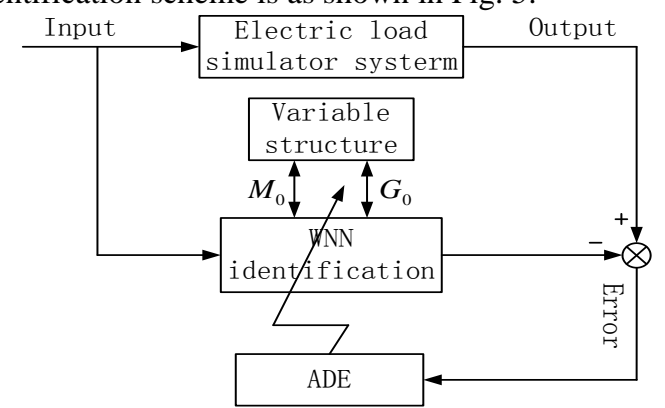

Fig. 5. The diagram of the identification scheme with ADE-VSWNN

\section{Simulation}

In order to further verify the validity and practicability of the methods above, a hardware- in-the-loop simulation of the ELS for GCSs is set up as shown in Fig. 1. The parameters of the actual system are as follows: the rotary inertia of the turret is $7000 \mathrm{~kg} \cdot \mathrm{m}^{2}$, the total friction torque $M_{f}$ is less than 1200 $\mathrm{N} \cdot \mathrm{m}$, and the center position of gravity is located in $0.35 \mathrm{~m}$ away from the rotation center of the turret, the reduction ratio is 360 . The maximum torque of the torque motor is $5.4 \mathrm{~N}$, the overload torque is $13.7 \mathrm{~N} \cdot \mathrm{m}$, the maximum speed is 6800 $\mathrm{r} / \mathrm{min}$, the rated power is $2.8 \mathrm{kw}$. Combining the relevant nominal value of the torque motor with the test results, the corresponding parameters of the ELS shown in Table 2. Considering the characteristics of the ELS system, the pseudo random multilevel and the linear frequency modulation signals are chosen as the input signals used for identification when the weight of the inertia disk is $2 \mathrm{~kg}$. The following simulations are carried out in an Inter core i5 CPU with $3.2 \mathrm{GHz}$ rate, $4 \mathrm{~GB}$ RAM and 64 bit operating system. In general, the parameters are self-adaptive from the global optional value offline, which greatly reduces the learning time. Owing to the constraint of the control period, the global optimum is not achieved only in a period. If the optimized parameters are not changed in 5 periods, these parameters are set as the fixed values. In the verification stage, the parameters are applied to evaluate the effectiveness of the proposed algorithm. For each simulation, twenty runs are made and the mean values, best mean values and standard derivations of mean absolute error (MAE), mean absolute percent error (MAPE), root mean square error (RMSE) and convergence epochs are shown in the following tables [48]. In addition, the position motor is working at a constant speed and a sinusoidal motion, respectively. The identification accuracy of the algorithm is evaluated through the test results. The ideal values of MAE, MAPE and RMSE are 0,0 and 0, respectively, when the global optimum of the fitness objective function $f_{\text {best }}$ is set 0.999 , and the value of RMSE is chosen as 0.001 . 
Table 2 The parameters of the ELS for the GCS

\begin{tabular}{ccc}
\hline Symbol & Value & Unit \\
\hline$K_{r}$ & 2.8 & $\mathrm{~V} / \mathrm{A}$ \\
$K_{t}$ & 2.6 & $\mathrm{~N} \cdot \mathrm{m} / \mathrm{A}$ \\
$K_{e}$ & 29.6 & $\mathrm{~V} / \mathrm{krpm}$ \\
$K_{u}$ & 2.6 & $\mathrm{~V} /(\mathrm{N} \cdot \mathrm{m})$ \\
$K_{i}$ & 15.2 & $\mathrm{~A} / \mathrm{V}$ \\
$K_{l}$ & 1 & $\mathrm{~V} / \mathrm{A}$ \\
$G_{f}$ & 0.72 & $\mathrm{~N} \cdot \mathrm{m} /^{\circ}$ \\
$L_{m}$ & 0.0036 & $\mathrm{H}$ \\
$R_{m}$ & 1.2 & $\Omega$ \\
$J_{m}$ & 0.038 & $\mathrm{~kg} \cdot \mathrm{m}^{2}$ \\
$B_{m}$ & 0.22 & $\mathrm{~N} \cdot \mathrm{m} \cdot \mathrm{s} / \mathrm{rad}$ \\
$h$ & 12 & $\mathrm{~kg} \cdot \mathrm{m}^{2}$ \\
$J_{i}$ & 0.0049 & \\
$K_{j}$ & 24 & \\
\hline & &
\end{tabular}

\subsection{The input with pseudo random multilevel signal}

In the pseudo random multilevel signal (PRMS) [49], also known as the pseudo random binary sequence (PRBS) of an amplitude-modulated (AM) signal, the amplitude will vary irregularly along the time dimension. The pseudo random multilevel signal is input to the ELS, while the selection of the input signal $T_{d}$ is from $-5 \mathrm{~N}$ to $+5 \mathrm{~N}$, which produces the corresponding control voltage across the controller, and $T_{g}$ is the output of the torque sensor shown in Fig. 6. The sample period is $2 \mathrm{~ms}$, the number of outputs is 5000 . The position motor is working at a constant speed.

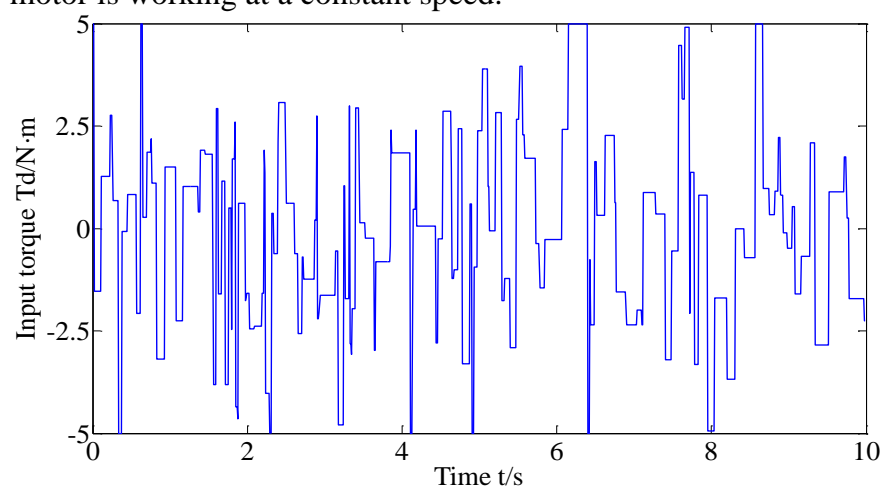

(b) The input data

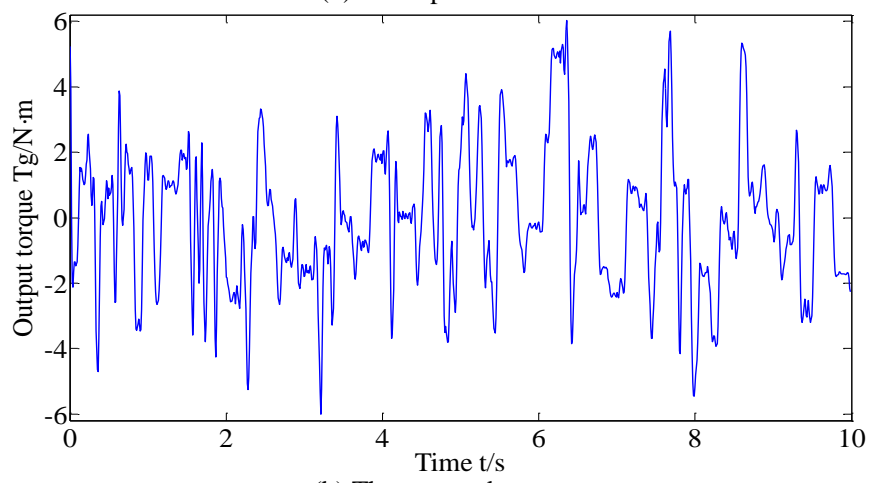

(b) The output data

Fig. 6. The input and output of PRMS

With respect to the input and output of Fig. 7, the former 2500 data items within $5 \mathrm{~s}$ are used for training, while the latter 2500 data items within $5 \mathrm{~s}$ are used for verification. The actual and expected outputs of WNN, ADE-WNN and ADE-VSWNN are shown in Fig. 7-9. The convergence epochs are shown in Fig. 10.

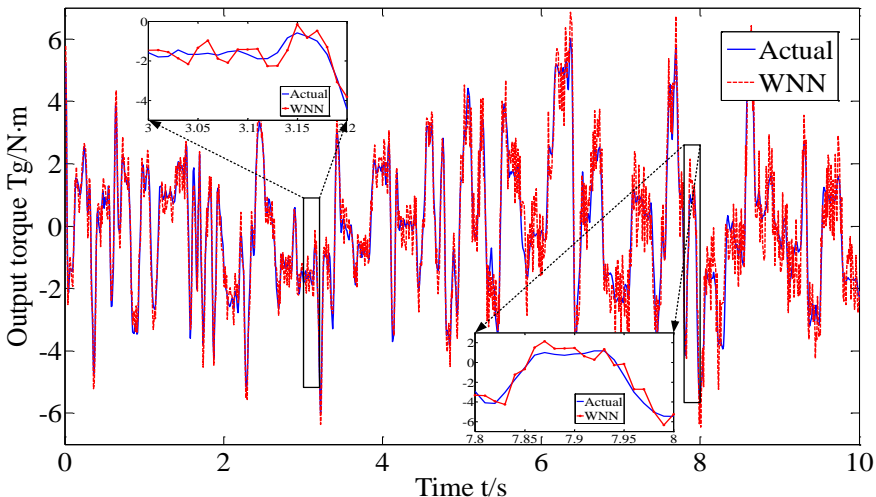

Fig. 7. The identification output of WNN with PRMS

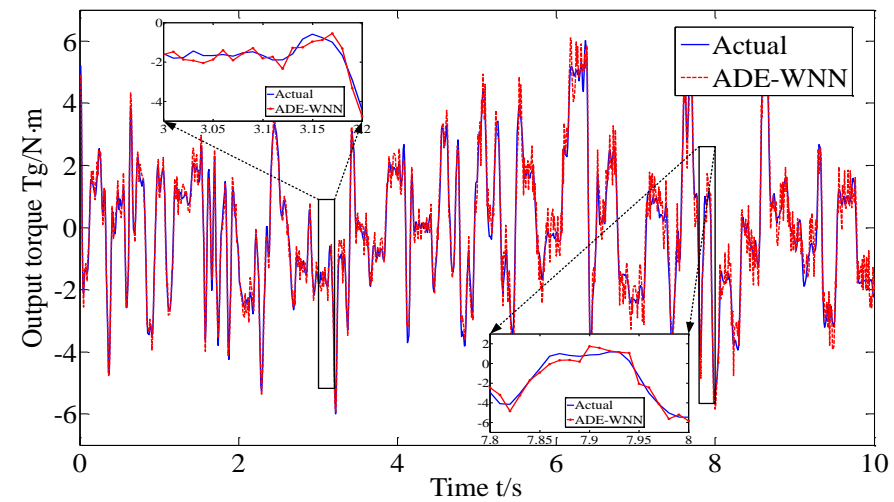

Fig. 8. The identification output of ADE-WNN with PRMS

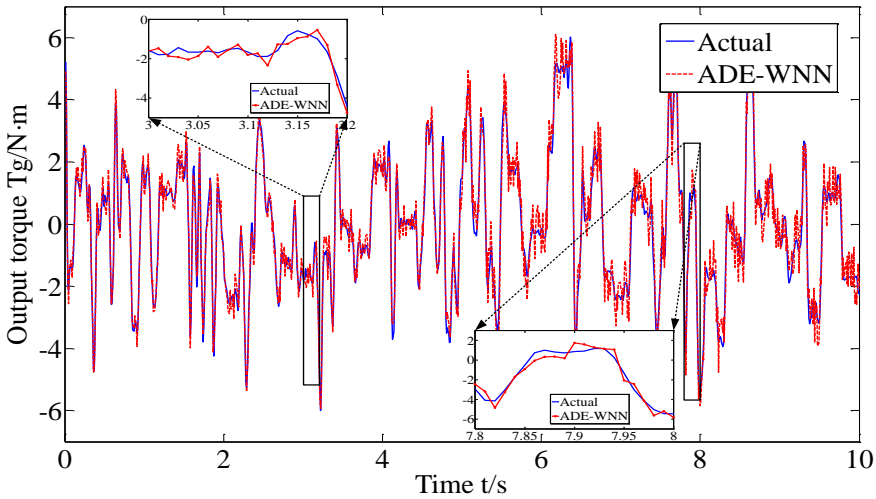

Fig. 9. The identification output of ADE-VSWNN with PRMS

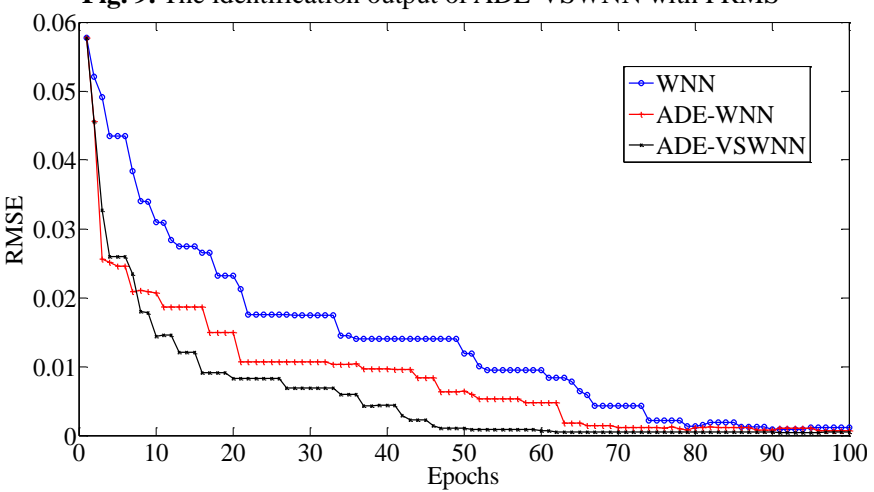

Fig. 10. The convergence epochs of identification with PRMS

In order to compare the approximation degree of different identification methods and the modeling precision of different models, the following precision indexes are defined, chosen depending on the specific situation of the different models:

(1) Mean absolute error (MAE)

$\operatorname{MAE}(y, \hat{y})=\frac{1}{\mathrm{n}} \sum_{i=1}^{\mathrm{n}}|y(i)-\hat{y}(i)|$ 
(2) Mean absolute percent error(MAPE)

$\operatorname{MAPE}(y, \hat{y})=\frac{1}{\mathrm{n}} \sum_{i=1}^{\mathrm{n}} \frac{|y(i)-\hat{y}(i)|}{|y(i)|} \quad y(i) \neq 0$.

(3) Root mean square error(RMSE)

$\operatorname{RMSE}(y, \hat{y})=\sqrt{\frac{1}{\mathrm{n}} \sum_{i=1}^{\mathrm{n}}[y(i)-\hat{y}(i)]^{2}}$

where $y$ is the output torque of the actual system; $\hat{y}$ is the output torque of the identification system; the MAPE tends to be distorted because of the strong negative impact; RMSE is used to measure the precision of the model. The smaller the calculated value, the closer the representation model is to the actual system. Table 3 shows that the training time online with
PRMS based on offline learning is described, which is 2 minutes faster than only offline learning. Table 4 further summarizes the t-test results with PRMS to test the significance of the difference in different methods, which relies on the mean, best mean and standard derivation values of the MAE, MAPE, RMSE and convergence epochs. Note: Numbers are statistically significant at 0.05 in two-sided tests.

Table 3

The comparison of the training time with PRMS

\begin{tabular}{cccc}
\hline $\begin{array}{c}\text { The verification } \\
\text { method }\end{array}$ & WNN & ADE-WNN & $\begin{array}{c}\text { ADE- } \\
\text { VSWNN }\end{array}$ \\
\hline $\begin{array}{c}\text { The mean training } \\
\text { time (ms) }\end{array}$ & 71.8 & 46.3 & 25.6 \\
\hline
\end{tabular}

Table 4

The t-test results with PRMS

(a)

\begin{tabular}{ccccccc}
\hline \multirow{2}{*}{ WNN Index } & \multicolumn{3}{c}{ Training } & & Verification \\
\cline { 2 - 7 } & Mean & Best mean & Std & Mean & Best mean & Std \\
MAE & 0.3448 & 0.1187 & 0.1452 & 0.5181 & 0.1273 & 0.2489 \\
MAPE & 0.4009 & 0.2273 & 0.1633 & 0.5790 & 0.1978 & 0.2539 \\
RMSE & 0.3963 & 0.1846 & 0.1362 & 0.5239 & 0.1976 & 0.2485 \\
Epochs & 93.25 & 82.0 & 7.7608 & - & - & - \\
\hline
\end{tabular}

(b)

\begin{tabular}{|c|c|c|c|c|c|c|}
\hline \multirow{2}{*}{$\begin{array}{l}\text { ADE-WNN } \\
\text { Index }\end{array}$} & \multicolumn{3}{|c|}{ Training } & \multicolumn{3}{|c|}{ Verification } \\
\hline & Mean & Best mean & Std & Mean & Best mean & Std \\
\hline MAE & 0.2580 & 0.0937 & 0.1204 & 0.4070 & 0.1063 & 0.1673 \\
\hline MAPE & 0.3670 & 0.1692 & 0.1077 & 0.4707 & 0.1725 & 0.2014 \\
\hline RMSE & 0.3340 & 0.1182 & 0.1193 & 0.4397 & 0.1397 & 0.1930 \\
\hline Epochs & 71.45 & 63.0 & 5.2172 & - & - & - \\
\hline
\end{tabular}

\begin{tabular}{ccccccc}
\multicolumn{1}{l}{ (c) } & \multicolumn{3}{c}{} & \multicolumn{3}{c}{ Verification } \\
\hline ADE-VSWNN & Training & Mean & Best mean & Std \\
\cline { 2 - 6 } Index & Mean & Best mean & Std & 0.2352 & 0.0895 & 0.0936 \\
MAE & 0.1396 & 0.0539 & 0.0715 & 0.1265 & 0.0611 & 0.0532 \\
MAPE & 0.0902 & 0.0502 & 0.0426 & 0.2555 & 0.1027 & 0.0826 \\
RMSE & 0.1544 & 0.0795 & 0.0510 & - & - & - \\
Epochs & 48.15 & 40.0 & 3.9417 & &
\end{tabular}

(d)

\begin{tabular}{cccccc}
\hline \multirow{2}{*}{ Index } & \multicolumn{3}{c}{ t-values } \\
\cline { 2 - 5 } & \multicolumn{2}{c}{ WNN and ADE-WNN } & \multicolumn{2}{c}{ WNN and ADE-VSWNN } & ADE-WNN and ADE-VSWNN \\
\cline { 2 - 5 } & Training & Verification & Training & Verification & Training \\
\hline MAE & 1.8112 & 2.0508 & 2.9211 & 2.3655 & 1.5659 \\
MAPE & 2.0718 & 1.7885 & 3.3180 & 3.2552 & 1.1254 \\
RMSE & 1.8611 & 1.9804 & 2.6528 & 3.0135 & 1.6053 \\
Epochs & 3.6737 & - & 5.5859 & 1.5979 & 2.5859 \\
\hline
\end{tabular}

The main conclusions from the above are as follows: the identification accuracy of the model with the WNN, ADE-WNN, ADE-VSWNN is gradually enhanced. The identification accuracy at the training stage is higher than that at the verification stage, which provides the high accuracy identification at the verification stage with strong support. All the indicators of ADE-VSWNN are greatly improved comparatively, and the number of the convergence epochs is the least, which reduces the computation complexity and improves the control ability in a real-time system. The values with the same absolute differences on one or the other side of the global optimum values are evaluated as opposite conclusions. Therefore, the t-test results might be misleading and successful rate is a better measure if reaching the global optimum is very important. In summary, the Table 4 further shows that the ADE-VSWNN with great significance is statistically better than the WNN and ADE-VSWNN.

\subsection{The input with linear frequency modulation signal}

The linear frequency-modulation signal is Chrip, known as multiple sinusoidal signal, which consists of a series of increasing frequency sine signal. The selection of the input signal follows [27]

$$
T_{d}(i)=T_{d 0}+\alpha \cdot \sin \left(\omega_{i} i T_{0}\right) \quad i=1,2, \ldots, n .
$$

where $\omega_{i}=\omega_{\text {start }}+\left(\omega_{\text {end }}-\omega_{\text {start }}\right) i / n, \omega_{\text {start }}$ is the start frequency, $\omega_{\text {end }}$ is the end frequency; $T_{0}$ is the sample time. The signal is used as the excitation signal of the ELS, which should be the combination of a series of different offsets $T_{d 0}$ and gains $\alpha$. The input and output curves are shown in Fig. 12 when the position motor is working at constant speed, the start frequency $\omega_{\text {start }}$ is $1 \mathrm{rad} / \mathrm{s}$, the end frequency $\omega_{\text {end }}$ is 20 $\mathrm{rad} / \mathrm{s}$, the sample time $T_{0}$ is $2 \mathrm{~ms}$, the offset $T_{d 0}$ is 0 , the gain $\alpha$ is 5.0, and the number of samples is 5000. The input and output curve are shown in the figure below when the position motor is working at a constant speed. 


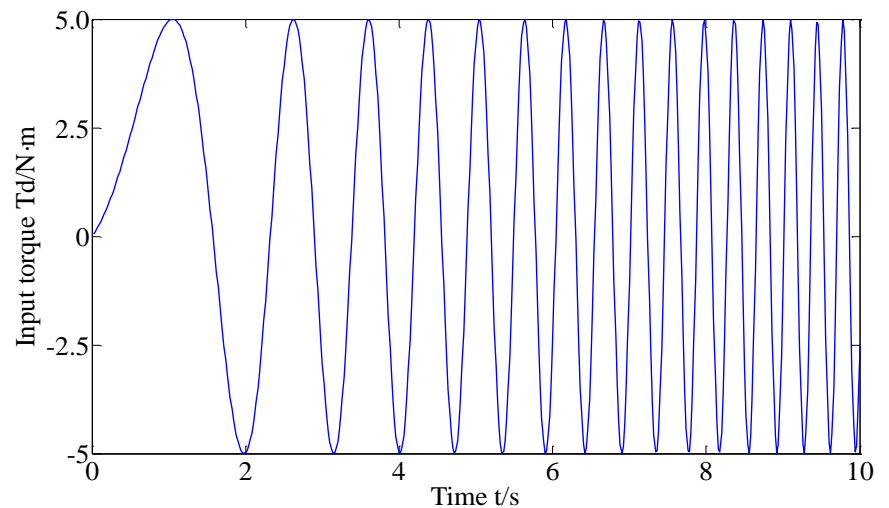

(a) The input data

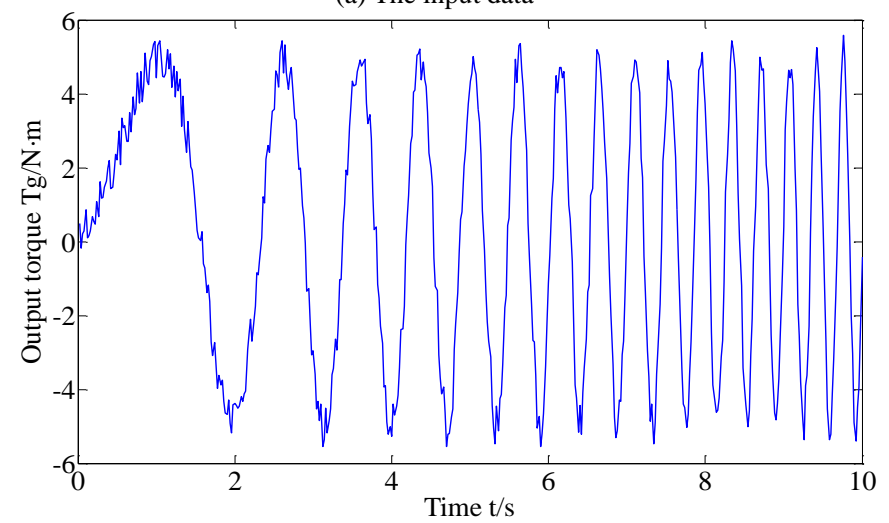

(b) The output data

Fig. 11. The input and output of Chrip in constant speed

Considering the inputs and outputs in Fig. 12, the former 2500 data items within $5 \mathrm{~s}$ are used for training, the latter 2500 data items within $5 \mathrm{~s}$ are used for verification. The actual and expected outputs of WNN, ADE-WNN and ADE-VSWNN are shown in Fig. 12-17, while the convergence epochs are shown in Fig. 18. The number of neurons in the ADE-VSWNN is shown in Fig. 19.

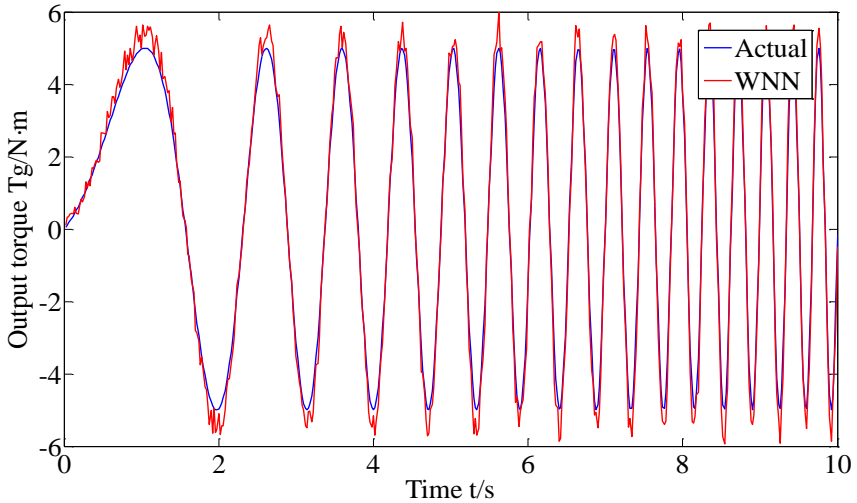

Fig. 12. The output of WNN of Chrip in constant speed

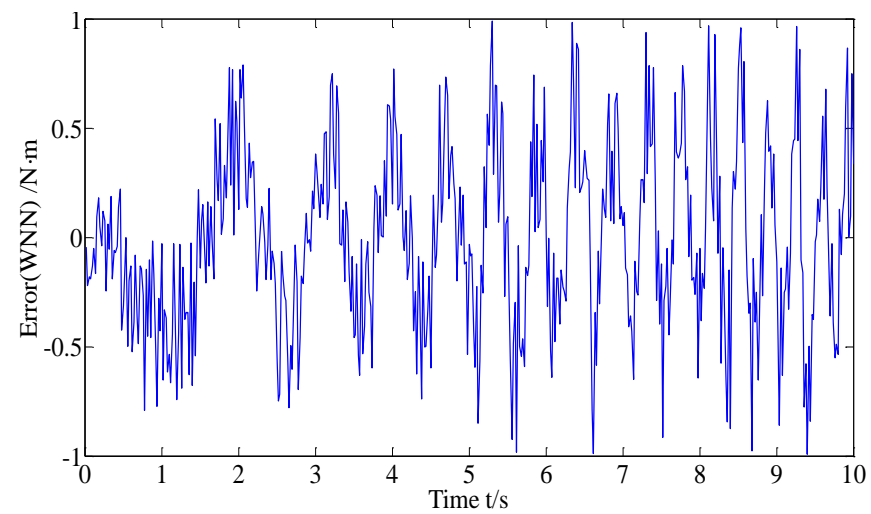

Fig. 13. The output error of WNN of Chrip in constant speed

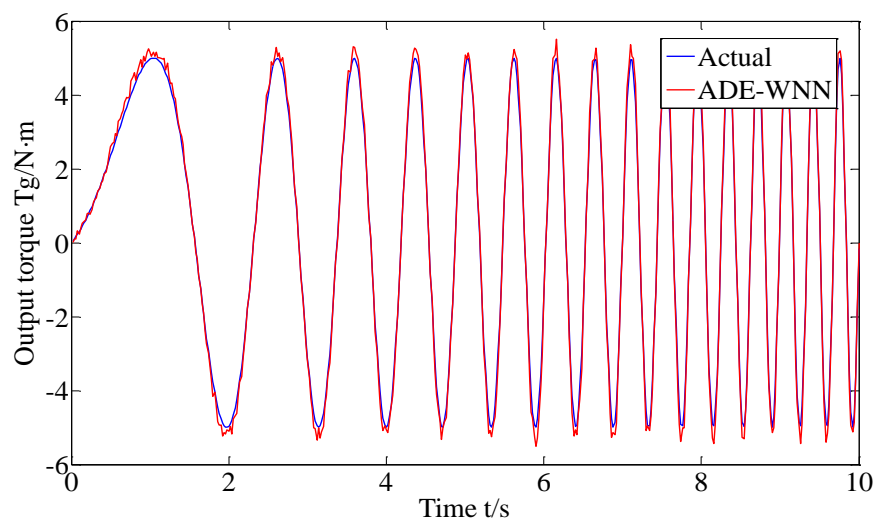

Fig. 14. The output of ADE-WNN of Chrip in constant speed

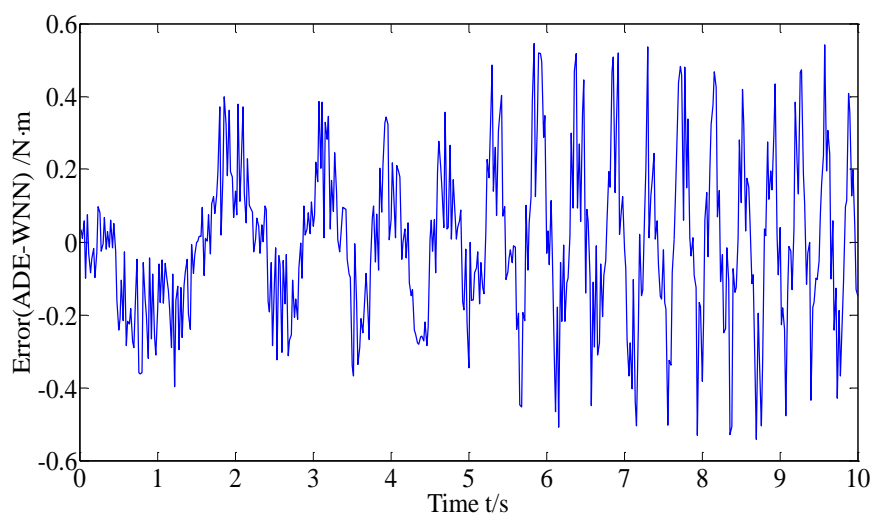

Fig. 15. The output error of ADE-WNN of Chrip in constant speed

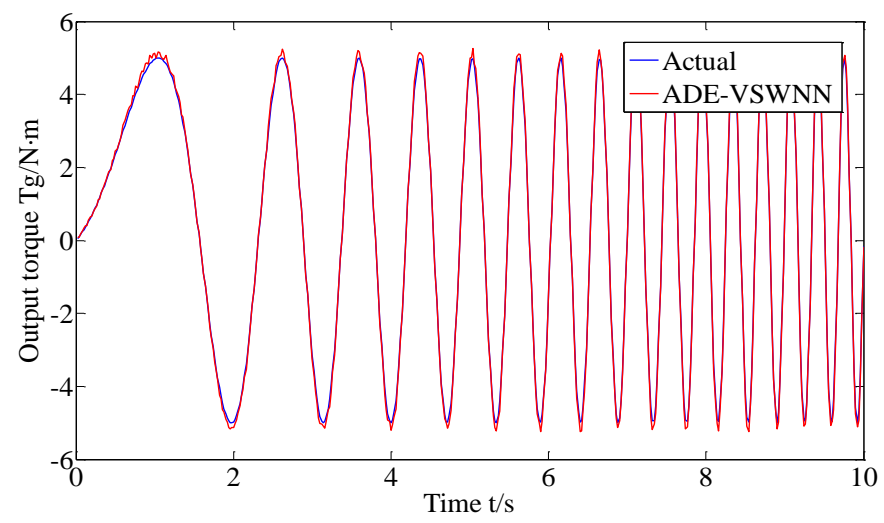

Fig. 16. The output of ADE-VSWNN of Chrip in constant speed

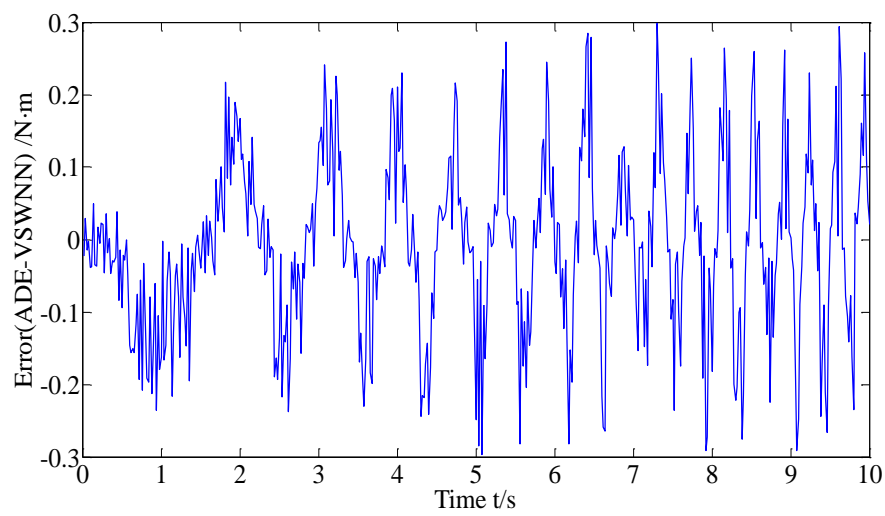

Fig. 17. The output error of ADE-VSWNN of Chrip in constant speed 


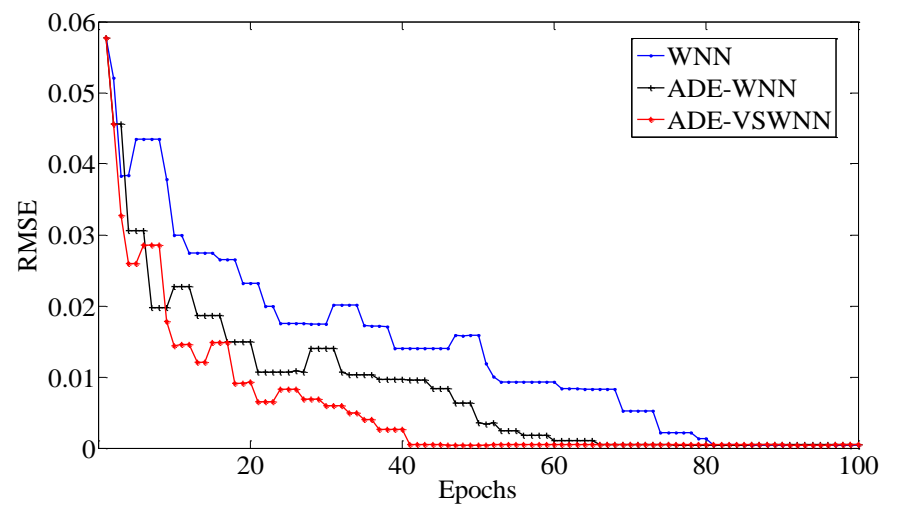

Fig. 18. The convergence epochs of Chrip in constant speed

Table 5

The comparison of the training time with Chrip

\begin{tabular}{cccc}
\hline $\begin{array}{c}\text { The verification } \\
\text { method }\end{array}$ & WNN & ADE-WNN & $\begin{array}{c}\text { ADE- } \\
\text { VSWNN }\end{array}$ \\
\hline $\begin{array}{c}\text { The mean training } \\
\text { time (ms) }\end{array}$ & 53.2 & 36.7 & 18.5 \\
\hline
\end{tabular}

Table 6

The t-test results with Chrip in constant speed

(a)

\begin{tabular}{ccccccc}
\hline WNN & \multicolumn{3}{c}{ Training } & & \multicolumn{3}{c}{ Verification } \\
\cline { 2 - 6 } Index & Mean & Best mean & Std & Mean & Best mean & Std \\
\hline MAE & 0.3249 & 0.1025 & 0.1684 & 0.5046 & 0.1209 & 0.2637 \\
MAPE & 0.1645 & 0.0863 & 0.0762 & 0.2418 & 0.0947 & 0.1083 \\
RMSE & 0.3538 & 0.1692 & 0.1298 & 0.4014 & 0.2082 & 0.1340 \\
Epochs & 80.45 & 68.0 & 6.9782 & - & - & - \\
\hline
\end{tabular}

(b)

\begin{tabular}{ccccccc}
\hline ADE-WNN & \multicolumn{3}{c}{ Training } & \multicolumn{3}{c}{ Verification } \\
\cline { 2 - 6 } Index & Mean & Best mean & Std & Mean & Best mean & Std \\
\hline MAE & 0.2406 & 0.0859 & 0.1082 & 0.3629 & 0.1867 & 0.1182 \\
MAPE & 0.1405 & 0.0652 & 0.0428 & 0.1862 & 0.0946 & 0.0569 \\
RMSE & 0.3029 & 0.1229 & 0.1092 & 0.3219 & 0.1228 & 0.1595 \\
Epochs & 65.15 & 57.0 & 4.3953 & - & - & - \\
\hline
\end{tabular}

(c)

\begin{tabular}{ccccccc}
\multicolumn{1}{l}{ (c) } & \multicolumn{3}{c}{ Training } & & \multicolumn{3}{c}{ Verification } \\
\cline { 2 - 6 } ADE-VSWNN & Mean & Best mean & Std & Mean & Best mean & Std \\
Index & 0.1202 & 0.0392 & 0.0642 & 0.1950 & 0.0703 & 0.0862 \\
MAE & 0.0994 & 0.0418 & 0.0398 & 0.1092 & 0.0439 & 0.0419 \\
MAPE & 0.1296 & 0.0562 & 0.0481 & 0.2047 & 0.0978 & 0.0752 \\
RMSE & 38.75 & 34.0 & 2.8935 & - & - & - \\
Epochs & & & & &
\end{tabular}

(d)

\begin{tabular}{cccccc}
\hline & \multicolumn{4}{c}{ t-values } \\
\cline { 2 - 6 } Index & \multicolumn{2}{c}{ WNN and ADE-WNN } & \multicolumn{2}{c}{ WNN and ADE-VSWNN } & ADE-WNN and ADE-VSWNN \\
\cline { 2 - 6 } & Training & Verification & Training & Verification & Training \\
nyAE & 1.6739 & 2.0025 & 2.6427 & 3.1274 & 1.3285 \\
MAPE & 1.8904 & 1.6582 & 3.1758 & 2.0438 & 1.0659 \\
RMSE & 1.6840 & 1.4416 & 2.3862 & 2.7841 & 1.3793 \\
Epochs & 3.0516 & - & 4.4294 & - & 2.1797 \\
\hline
\end{tabular}

The main conclusions above are as follows: The conclusion of section 5.1 is verified in section 5.2, which further shows the stability of the proposed algorithms. Because the input signal in section 5.2 has the periodicity property, the identification accuracy is relatively higher than section 5.1. When the torque motor changes the motor direction or the input signal frequency is bigger, the identification error is comparatively larger.
Using the analysis method of section 5.1, Table 5 shows the he training time online with Chrip based on offline learning is described, which are even better than that in the Table 3. Table 6 further summarizes the t-test results with Chrip in constant speed to test the significance of the difference in different methods, which relies on the mean, best mean and standard derivation values of the MAE, MAPE, RMSE, and convergence epochs. Note: Numbers are statistically significant at 0.05 in two-sided tests. 
the number reduces to the least value of 9. Due to the increase of the input signal frequency, the number of neurons is always more than 9. Also, when the torque motor changes the motor direction or the input signal frequency is bigger, the number of neurons is relatively larger. The number of neurons is greater than 11 after $8 \mathrm{~s}$, which means the computational complexity is higher.

The weight of the inertia disks is $2 \mathrm{~kg}$, which is the biggest rotational inertia converted to the inertia of the torque motor shaft. The parameters of linear frequency modulation signal are chosen as follows: the start frequency $\omega_{\text {start }}$ is $1 \mathrm{rad} / \mathrm{s}$; the end frequency is $20 \mathrm{rad} / \mathrm{s}$; the sample period $\mathrm{T}_{0}$ is $2 \mathrm{~ms}$; the offset $\mathrm{T}_{\mathrm{d} 0}$ is 0 , the gain $\alpha$ is 5.0 ; the number of samples is 5000. The input and output curves are shown in the figures below when the position motor working in sinusoidal motion, with $y(t)=2 \sin (0.5 \pi t)$. Table 7 further summarizes the t-test results with Chrip in sinusoidal motion to test the significance of the difference in different methods, which relies on the mean, best mean and standard derivation values of the MAE, MAPE, RMSE and convergence epochs. Note: Numbers are statistically significant at 0.025 in single-sided tests.

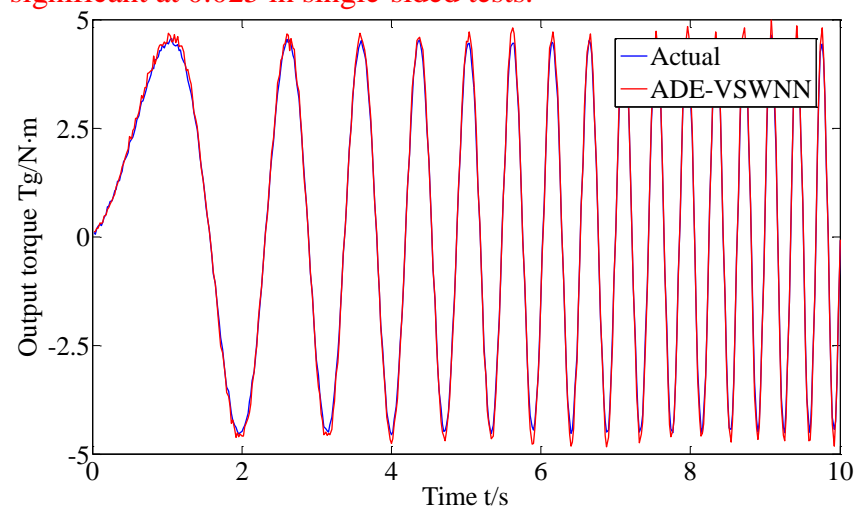

Fig. 20. The output of ADE-VSWNN of Chrip in sinusoidal motion

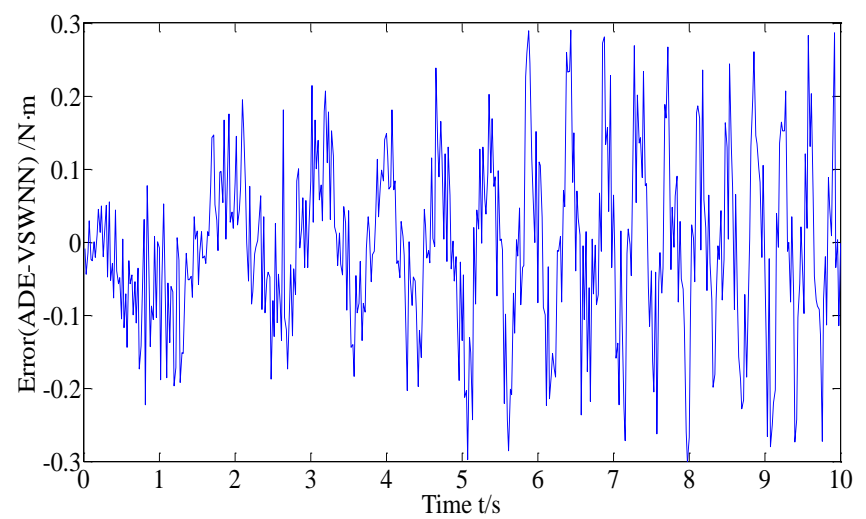

Fig. 21. The output error of ADE-VSWNN of Chrip in sinusoidal motion

Table 7

The t-test results with Chrip in sinusoidal motion

\begin{tabular}{|c|c|c|c|c|c|}
\hline \multicolumn{2}{|c|}{$\begin{array}{c}\text { ADE- } \\
\text { VSWNN }\end{array}$} & MAE & MAPE & RMSE & Epochs \\
\hline \multirow{4}{*}{ Training } & Mean & 0.1483 & 0.1328 & 0.1890 & 47.25 \\
\hline & $\begin{array}{l}\text { Best } \\
\text { mean }\end{array}$ & 0.0584 & 0.0694 & 0.0862 & 42.0 \\
\hline & Std & 0.0738 & 0.0592 & 0.0692 & 3.4855 \\
\hline & t-values & 1.6388 & 1.4175 & 1.7269 & 2.5389 \\
\hline \multirow{4}{*}{$\begin{array}{l}\text { Veri } \\
\text { fica } \\
\text { tion }\end{array}$} & Mean & 0.2294 & 0.1637 & 0.2571 & - \\
\hline & $\begin{array}{l}\text { Best } \\
\text { mean }\end{array}$ & 0.0859 & 0.0973 & 0.1003 & - \\
\hline & Std & 0.0983 & 0.0571 & 0.1184 & - \\
\hline & t-values & 1.9762 & 1.8364 & 2.0156 & - \\
\hline
\end{tabular}

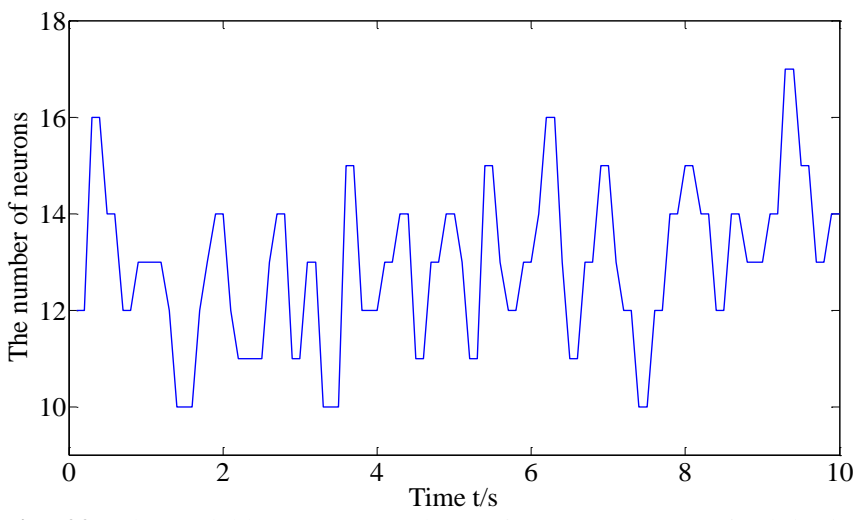

Fig. 22. The variable neuron numbers of ADE-VSWNN of Chrip in sinusoidal motion

As shown in Fig. 22, the number of neurons is 12 at the start stage, then the number increases to 16 along the time, and later the number reduces to the least value of 10 . Due to the increase of the input signal frequency, the number of neurons is always more than 9 . The surplus torque introduced by the inertia torque plays a great negative influence on the electric load simulator compared with the position motor working at a constant speed. In terms of the ADE-VSWNN, the computation complexity is higher, and the convergence speed is slower.

\section{Conclusion}

Taking into consideration the uncertainty and the strong nonlinearities in the ELS, this paper first introduces the composition and the principle of the ELS, then puts forward the identification strategy of the ADE-VSWNN, then sets up the hardware-in-the-loop simulation test platform of the ELS for GCSs. Finally, the pseudo random multilevel and linear frequency modulation signals are used as input when the position motor is working in a different state. When using the above algorithm, the following conclusions are achieved:

(1) First of all, the ADE-VSWNN identification strategy not only greatly reduces the static error existing in the system, but also is better than the WNN, ADE-WNN algorithm. Combined with a series of different forms of input signals in the experiments, the strong robustness and generality are further verified, which can be introduced to other nonlinear systems.

(2) The degree of the effect of the surplus torque varies with the frequency of the input. Although all results conform to the requirements for the technique indexes, the identification error is comparatively larger, and the computational complexity is higher when the torque motor changes the motor direction, or when the input signal frequency is bigger, which points out directions for future research.

\section{Acknowledgement}

The authors would like to express our gratitude to all anonymous reviewers for their valuable comments. This work was supported by the National Science Foundation of China under Grants 51305205.

\section{References}

[1] Y. X. Shang, H.Yuan, Z.X.Jiao, N.Yao.Matching design of hydraulic load simulator with aerocraft actuator, Chinese Journal of Aeronautics 26 (2) (2013) 470-480.

[2] J.Y. Yao, Z.X. Jiao, Y.X. Shang, C. Huang, Adaptive nonlinear optimal compensation control for electro-hydraulic load simulator, Chinese Journal of Aeronautics 23(1) (2010) 720-733.

[3] C.W. Wang, Z.X. Jiao, S. Wu, Y.X. Shang, An experimental study of the dual-loop control of electro-hydraulic load simulator (EHLS), Chinese Journal of Aeronautics 26(6) (2013) 1586-1595.

[4] C.W. Wang, Z.X.Jiao,S. Wu,Y.X.Shang, Nonlinear adaptive torque control of electro-hydraulic load system with external active motion 
disturbance, Mechatronics 24(1) (2014) 32-40.

[5] J.Y. Yao, Z.X. Jiao, B. Yao, Y.X. Shang, W.B. Dong, Nonlinear adaptive robust force control of hydraulic load simulator, Chinese Journal of Aeronautics 25(1) (2012) 766-775.

[6] C.W. Wang, Z.X. Jiao, S. Wu, Y.X. Shang, A practical nonlinear robust control approach of electro-hydraulic load simulator, Chinese Journal of Aeronautics 27(3) (2014)735- 744.

[7] L. Wang, L.F.Qian, Q. Gao, Q. Guo, Torque load simulator control research in servo system based on fuzzy PID with gray prediction, Acta Armamentarii 33(11) (2012) 101-104

[8] L. Wang, L.F. Qian,Q. Guo,Torque control of servo load simulator with generalized dynamic fuzzy neural network based on grey prediction, Proceedings of ICMEME2011 AMM (2012) 707-712.

[9] S. I.Han, K.S.Lee, Robust friction state observer and recurrent fuzzy neural network design for dynamic friction compensation with backstepping control, Mechatronics 20(3) (2010) 384-401.

[10] I.B.Tijani, R.Akmeliawati, Support vector regression based friction modeling and compensation in motion control system, Engineering Applications of Artificial Intelligence 25(5) (2012) 1043-1052

[11] J. Yao, Z.Jiao, B.Yao, Robust control for static loading of electro-hydraulic load simulator with friction compensation. Chinese Journal of Aeronautics 25(6) (2012)954-962.

[12] F. Giri, Y. Rochdi, A. Radouane,A. Brouri, , F. Z. Chaoui, Frequency identification of nonparametric Wiener systems containing backlash nonlinearities, Automatica 49(1) (2013) 124-137.

[13] H.Moradi, H.Salarieh, Analysis of nonlinear oscillations in spur gear pairs with approximated modelling of backlash nonlinearity, Mechanism and Machine Theory 51(1) (2012) 14-31.

[14] S.Y.Chen, J.Y.Tang, C.W.Luo, Q.B.Wang, Nonlinear dynamic characteristics of geared rotor bearing systems with dynamic backlash and friction, Mechanism and Machine Theory 46(4) (2011) 466-478

[15] S. H. Ling, P. P. San, K. Y. Chan, F. H. F. Leung, Y. Liu, An intelligent swarm based-wavelet neural network for affective mobile phone design, Neurocomputing 142(1) (2014) 30-38.

[16] F. Vavak, T. Fogarty, K. Jukes, A genetic algorithm with variable range of local search for tracking changing environments, in: Proceedings of the Fourth Conference on Parallel Problem Solving from Nature, 1996.

[17] J. Knowles, D. Corne, A comparative assessment of memetic, evolutionary and constructive algorithms for the multi-objective d-msat problem, in: Genetic and Evolutionary Computation Workshop Proceeding, 2001.

[18] N. Krasnogor, Self-generating metaheuristics in bioinformatics: the protein structure comparison case, in: Genetic Programm- ing and Evolvable Machines, Kluwer Academic Publishers, 5(1) (2004) 181-201.

[19] H. L. Wei, S. A. Billings, Y. F. Zhao, L. Z. Guo, An adaptive wavelet neural network for spatio-temporal system identification, Neural Networks 23(10) (2010) 1286-1299.

[20] F. Jahangiri, A. Doustmohammadi, M. B. Menhaj, An adaptive wavelet differential neural networks based identifier and its stability analysis, Neurocomputing 77(1) (2012)12-19.

[21] S.Ganjefar, M.Tofighi, Single-hidden-layer fuzzy recurrent wavelet neural network: Applications to function approximation and system identification Information Sciences, 294(10) (2015) 269-285.

[22] R. Cheng, Y. Bai, A novel approach to fuzzy wavelet neural network modeling and optimization, International Journal of Electrical Power \& Energy Systems 64(0) (2015) 671-678.

[23] C. M. Lin, C. S. Hsueh, C. H. Chen, Robust adaptive backstepping control for a class of nonlinear systems using recurrent wavelet neural network, Neurocomputing 142(1) (2014) 372- 382.

[24] H. Y. Chen, J. W. Liang, Piezoelectric-actuated drop-on-demand droplet generator control using adaptive wavelet neural network controller, Journal of Process Control 24(5) (2014) 578-585.

[25] C. F. Hsu, Adaptive neural complementary sliding-mode control via functional-linked wavelet neural network, Engineering Applications of Artificial Intelligence 26(4) (2013) 1221-1229.

[26] M. Alizadeh, M.Tofighib, Full-adaptive THEN-part equipped fuzzy wavelet neural controller design of FACTS devices to suppress inter-area oscillations, Neurocomputing,118(22) (2013) 157-170

[27] B. Subudhi, D.Jena, Nonlinear system identification using memetic differential evolution trained neural networks, Neurocomputing,74(10) (2011) 1696-1709.

[28] P. P. San, S. H. Ling, H. T. Nguyen, Hybrid PSO-based variable translation wavelet neural network and its application to hypoglycemia detection system, Neural Computing and Applications 23(7) (2013) 2177-2184.

[29] C. F. Hsu, A self-evolving functional-linked wavelet neural network for control applications, Applied Soft Computing 13(11) (2013) 4392 4402.

[30] C. F. Hsu, Adaptive PI Hermite neural control for MIMO uncertain nonlinear systems, Applied Soft Computing 13(5) (2013) 2569-2576.

[31] C. F. Hsu, Adaptive backstepping Elman-based neural control for unknown nonlinear systems, Neurocomputing 136(6)(2014) 170-179.

[32] B. Guo, The system research of electric load simulator, Harbin Institute of Technology (2012)31-40.

[33] Z. S. Li, M. Y. Wang, A novel method for restraining the redundancy torque based on DFNN,Journal of Harbin Institute of Technology 44(10) (2012)79-83

[34] R. Wang, S. L. Chen. Analysis of influence factors on output moment of electrical lord simulator, Journal of Test and Meas-urement Technology25(1)(2011)47-51.

[35] C.Canudas de Wit, H.Olsson, K.J. Astrom,P. Lischinsky,A new model for control of systems with friction, IEEE Transactions on Automatic Control 40(1)(1995) 419-425.

[36] A.H.Brain, D.Pierre, C.D.W.Carlos. A survey of models, analysis tools and compensation methods for the control of machines with friction, Automatica 30(7)(1994) 1083-1138.

[37] C.D.W.Carlos, P.Lischinsky, Adaptive friction compensation with partially known dynamic friction model, International Journal of Adaptive Control and Signal Processing 11(1)(1997) 65-80.

[38] M.Hamed, S.Hassan, Analysis of nonlinear oscillations in spur gea pairs with approximated modelling of backlash nonlinearity, Mechanism and Machine Theory 51(5)(2012) 14-31.

[39] R.Mezouki,J.A.Davila, L.Fridman, Backlash phenomenon observation and identification in electromechanical systems, Control Engineering Practice 15(4)(2007)447-457.

[40] L. Zhang, D.H.Zhang, Y.X.Su, C.Wang, Head pose estimation based on feature extraction, fuzzy C-means and neural network for driver assistance system, 11th IEEE International Conference on Control \& Automation (ICCA) (2004)677-682.

[41] L. Zhang, W. Zhou, L. Jiao, Wavelet support vector machine, IEEE Transactions on Systems, Man, and Cybernetics-Part B: Cybernetics 34 (1) (2004) 34-38.

[42] B. Liu, L.Wang, Y.H.Jin, Advances in differential evolution, Control and Decision22(7) (2007) 721-729.

[43] T. Warren Liao, R.J. Kuo, J.T.L. Hu, Hybrid ant colony optimization algorithms for mixed discrete-continuous optimization problems, Applied Mathematics and Computation 219(6) (2012) 3241-3252.

[44] H. Z. Yi, Q. L. Duan, T. W. Liao, Three improved hybrid metaheuristic algorithms for engineering design optimization, Applied Soft Computing 13 (5) (2013) 2433-2444.

[45] T. W. Liao, Two hybrid differential evolution algorithms for engineering design optimization, Applied Soft Computing 10 (4) (2010) 1188-1199.

[46] M. Mahdavi, M. Fesanghary, E. Damangir, An improved harmony search algorithm for solving optimization problems, Applied Mathematics and Computation 188(2) (2007) 1567-1579.

[47] C.M.Lin, H.Y.Lee, Self-organizing adaptive wavelet CMAC backstepping control system design for nonlinear chaotic systems, Nonlinear Analysis Real World Applications 14(1) (2013)206-223.

[48] X.S.Deng, X.Z.Wang, Incremental learning of dynamic fuzzy neural networks for accurate system modeling, Fuzzy Sets and Systems 160 (2009) 972-987

[49] R.Haber, Kevicaky, Nonlinear System Identification-Input-Output Modelling Approach, vola 1-2. Kluwer. 
Abstract: Owing to the complex nonlinearities of the electric load simulator (ELS) for the gun control system (GCS), the surplus torque plays a great negative impact on the performance of the loading system. This paper proposes a variable-structure wavelet-neural-network (VSWNN) identification strategy based on adaptive differential evolution (ADE). First of all, a mathematical model is established based on the structure and the working principle of the ELS. Then an intelligent identification method is applied, where the wavelet function is chosen as the excitation function, which improves the generalization and approximation ability of the neural network. The ADE is used to optimize the parameters, which solves the difficulty of determining the structure of the WNN. In order to reduce the computation complexity and speed up the convergence of the identification system, the adaptive laws of the pitch adjusting rate (PAR), band width (BW) and variable numbers of neurons are proposed. Finally, a pseudo random multilevel signal and a linear frequency modulation signal are chosen as input signals for the hardware-in-the-loop simulation. The test results show that the proposed ADE-VSWNN algorithm has superior validity and practicability, especially when the identification algorithm is used in the working circumstances with different inertial torque. Further, the high precision and strong robustness of the identification algorithm are further verified.

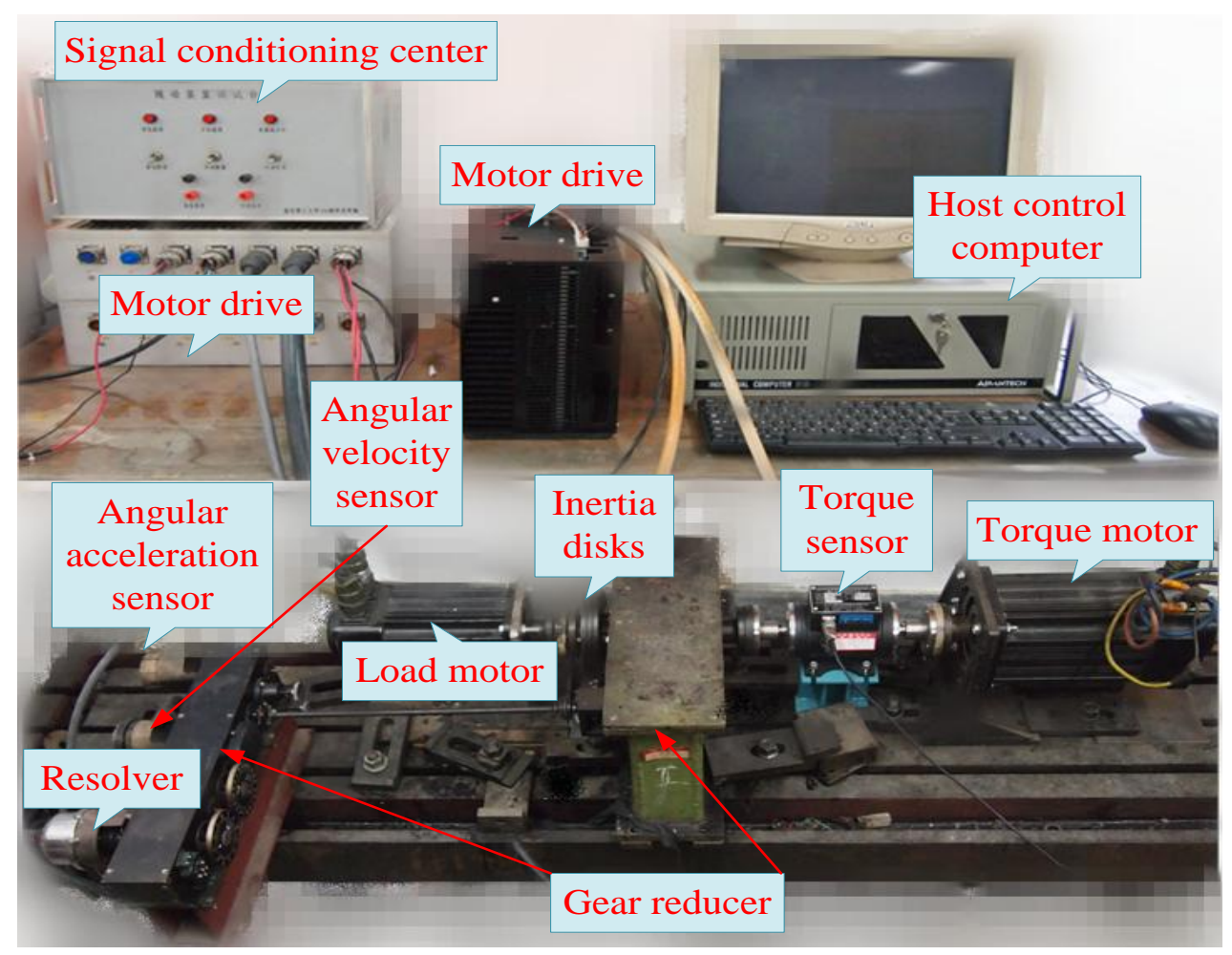

Fig. 1. The composition diagram of the ELS for GCSs 